\title{
Particle acceleration by large-amplitude waves revisited
}

\author{
R. PAKTER, $\dagger$ G. CORSO $\dagger$ and F. B. RIZZATO \\ Instituto de Física, Universidade Federal do Rio Grande do Sul, PO Box 15051, \\ 91501-970 Porto Alegre, Rio Grande do Sul, Brasil
}

(Received 24 May 1996 and in revised form 1 July 1996)

We reconsider the problem of particle acceleration by large-amplitude electromagnetic waves. We make use of a fully relativistic Hamiltonian formalism to show that, as opposed to nonelativistic results obtained by Kuo and Lee, acceleration in unmagnetized systems is severely arrested when the phase velocity of the electromagnetic mode approaches the speed of light. For subluminal waves, however, acceleration is shown to be still effective.

\section{Introduction}

With the advent of powerful radiation-generation systems such as free-electron lasers, cyclotron autoresonance masers, gyrotrons and ion-channel lasers, a good deal of effort has been directed to the study of the interaction of low-energy particles and large-amplitude electromagnetic waves (Sprangle et al. 1983; Davidson et al. 1989; Chen 1992; Pakter et al. 1993, 1994a, b; Friedland 1994; Kuo and Lee 1994). Whenever wave-particle exchange is likely to occur, particles can be strongly accelerated, a feature of importance not only for particle acceleration itself, but also for the current-drive techniques of controlled thermonuclear research.

Theoretical models have been developed in order to investigate the dynamics of charged particles, either magnetized or not, in intense electromagnetic fields. In particular, Kuo and Lee (1994) have analysed the interaction of accelerating singleparticles with a strong circularly polarized electromagnetic wave in the absence of a background magnetic field. Based on a Lorentz-force non-relativistic formalism, they have derived a nonlinear Schrödinger equation governing the time evolution of a single particle's parallel (with respect to the wave propagation vector) velocity. This equation has either periodic or soliton-like solutions, depending on the initial conditions. In the periodic case particles draw energy from the wave in a reversible way, accelerating and decelerating periodically. On the other hand, soliton-like acceleration has been found to be more efficient, with the velocity monotonically increasing up to extremely large values.

In the present work we perform a fully relativistic analysis of the problem. It is found that soliton-like solutions with finite wave amplitudes may only appear in those cases where the phase velocity of the wave, $v_{\phi}$, is smaller than $c$, the speed of light; in deep contrast to the non-relativistic results obtained by Kuo and Lee

$\dagger$ Present address: Instituto de Física, Universidade de São Paulo, PO Box 66318, 05389-970 São Paulo, Brasil. 
(1994), as $v_{\phi} \rightarrow c$ the wave amplitude necessary to drive soliton-like acceleration tends to infinity. At any rate, for $v_{\phi}<c$ the soliton-like acceleration is shown to be of relevance. In fact, it is shown that for a given and finite amplitude of the wave, there exists a unique value of $v_{\phi}<c$ that produces maximum acceleration, and that this unique value of $v_{\phi}$ is closely related to the one respective to the soliton-like process.

In view of the necessary condition $v_{\phi}<c$, we need a medium that decelerates the relevant electromagnetic modes. As shown experimentally by Von Laven et al. (1982), the deceleration can be obtained if one places the system in a dielectric medium whose refractive index is larger than unity. The resulting energization process could be loosely seen as an inverse Čerenkov effect where particles gain energy from a resonant wave propagating with phase velocity smaller than the speed of light.

To perform the calculations, we adopt a Hamiltonian approach. This seems to provide an easier way to look at the problem than the Lorentz-force formalism previously employed (Kuo and Lee, 1994).

The work is organized as follows. In Sec. 2 we introduce the model used in the present investigation; in Sec. 3 we analyse the main characteristics of the relativistic wave-particle dynamics, taking into account both solitary and periodic solutions; in Sec. 4 final discussions on the acceleration method described in the paper are presented.

\section{The model}

In the model we consider charged particles interacting with an intense right-hand circularly polarized electromagnetic wave of frequency $\omega$ and wave vector $\mathbf{k}=k \hat{\mathbf{z}}$. The vector potential of the wave, $\mathbf{A}$, is written as

$$
\mathbf{A}=\frac{2 \epsilon c}{\omega}[\sin (k z-\omega t) \hat{\mathbf{x}}+\cos (k z-\omega t) \hat{\mathbf{y}}],
$$

where $\epsilon$ is the wave electric field amplitude and $c$ is the speed of light; the phase velocity of the wave, $v_{\phi}$, is obtained as $v_{\phi} \equiv \omega / k$.

Scaling time and distance to $\omega$ and $\omega / c$ respectively the dimensionless singleparticle Hamiltonian turns out to be given by

$$
H=\left\{1+\left[P_{x}-\epsilon \sin \left(\frac{z}{f}-t\right)\right]^{2}+\left[P_{y}-\epsilon \cos \left(\frac{z}{f}-t\right)\right]^{2}+P_{z}^{2}\right\}^{1 / 2},
$$

where $H$ is normalized to $m_{o} c^{2}, \mathbf{P}$ to $m_{0} c$ and $\epsilon$ to $2 e / \omega m_{0} c$ with $e$ and $m_{0}$ the electron charge and rest mass, and $f \equiv \omega / c k=v_{\phi} / c$.

Performing a time-removal canonical transformation, $P_{z} \rightarrow P_{z}, z-f t \rightarrow \phi$ and $H \rightarrow \mathcal{H} \equiv H-f P_{z}$, we can write the new Hamiltonian as

$$
\mathcal{H}=\left\{1+\left[P_{x}-\epsilon \sin \left(\frac{\phi}{f}\right)\right]^{2}+\left[P_{y}-\epsilon \cos \left(\frac{\phi}{f}\right)\right]^{2}+\left(P_{z}\right)^{2}\right\}^{1 / 2}-f P_{z} \equiv \gamma-f P_{z}
$$

where $\gamma$ is introduced as the relativistic factor.

From the Hamiltonian, we can readily note that $P_{x}$ and $P_{y}$, the transverse canonical momenta, and $\mathcal{H}$ are conserved quantities. Writing the kinetic momenta as 
$p_{x}=p_{\perp} \cos \theta$ and $p_{y}=p_{\perp} \sin \theta$ and using the canonical relationship between $\mathbf{P}$ and p, we have

$$
\begin{aligned}
& P_{x}=p_{\perp, 0} \cos \theta_{0}+\epsilon \sin \left(\phi_{0} / f\right), \\
& P_{y}=p_{\perp, 0} \sin \theta_{0}+\epsilon \cos \left(\phi_{0} / f\right),
\end{aligned}
$$

where subscript ' 0 ' here means initial conditions. Then the Hamiltonian (2.3) can be cast into the generic form

$$
\begin{aligned}
\mathcal{H}= & \left\{1+p_{\perp, 0}^{2}+2 \epsilon^{2}\left[1-\cos \left(\frac{\phi-\phi_{0}}{f}\right)\right]\right. \\
& \left.+2 \epsilon p_{\perp, 0}\left[\sin \left(\frac{\phi}{f}+\theta_{0}\right)+\sin \left(\frac{\phi_{0}}{f}+\theta_{0}\right)\right]+\left(P_{z}\right)^{2}\right\}^{1 / 2}-f P_{z} \\
= & \gamma-f P_{z} .
\end{aligned}
$$

In the present paper we are mainly interested in the wave-particle interaction leading to particle acceleration. Therefore, as is typical in accelerating configurations, we consider particles with very low initial energies, $\mathbf{p}_{\perp, 0}=0$. The Hamiltonian system corresponding to this situation is

$$
\mathcal{H}=\left[1+2 \epsilon^{2}(1-\cos \psi)+\left(P_{z}\right)^{2}\right]^{1 / 2}-f P_{z}=\gamma-f P_{z},
$$

with

$$
\begin{gathered}
\dot{P}_{z}=-\frac{\partial \mathcal{H}}{\partial \phi}=-\frac{\epsilon^{2} \sin \psi}{f \gamma}, \\
\dot{\phi}=\frac{\partial \mathcal{H}}{\partial P_{z}}=\frac{P_{z}}{\gamma}-f,
\end{gathered}
$$

where the dot stands for time derivative, and $\left(\phi-\phi_{0}\right) / f \equiv \psi$.

\section{Acceleration process}

\subsection{Soliton-like acceleration}

Before we start the investigation of the relativistic dynamics dictated by the Hamiltonian (2.5), let us briefly analyse the soliton-like solutions obtained within the nonrelativistic regime studied by Kuo and Lee (1994). Expanding (2.5) for $\epsilon, P_{z} \ll 1$, one writes down an expression for the non-relativistic Hamiltonian $\mathcal{H}_{\mathrm{nr}}$ as a function of the coordinates $\psi_{\mathrm{nr}}$ and $v_{z, \mathrm{nr}}=P_{z, \mathrm{nr}}$ :

$$
\mathcal{H}_{\mathrm{nr}}=\frac{1}{2}\left(v_{z, \mathrm{nr}}-f\right)^{2}-\epsilon^{2} \cos \psi_{\mathrm{nr}} .
$$

We readily note that the above Hamiltonian is pendulum-like, with unstable fixed points located at $v_{z, \mathrm{nr}}^{u}=f$ and $\psi_{\mathrm{nr}}^{u}= \pm \pi$. The separatrix orbit joining these unstable fixed points and touching the $v_{z, n r}=0$ axis is precisely the curve along which the solitary solution found by Kuo and Lee (1994) evolves (see Fig. 1 - the separatrix is the thick curve). Along the separatrix, particles are accelerated monotonically from $v_{z, \mathrm{nr}, 0}=0$ and $\psi_{\mathrm{nr}, 0}=0$ up to $v_{z, \mathrm{nr}, \max }=1$ and $\psi_{n r}=-\pi$ for $f=1$ and $t \rightarrow \infty$ (in the generic case the maximum velocity satisfies $v_{z, \mathrm{nr}, \max }=v_{z, \mathrm{nr}}^{u}=f$ ). A major drawback of this kind of solution is that the smallness conditions imposed on $P_{z}$ and $\epsilon$ are not satisfied when $v_{z} \approx 1$. We shall proceed to cure this failure with the appropriate relativistic analysis. Before that, however, we remark that the numerical value of the non-relativistic Hamiltonian along the separatrix is $\epsilon^{2}$. 


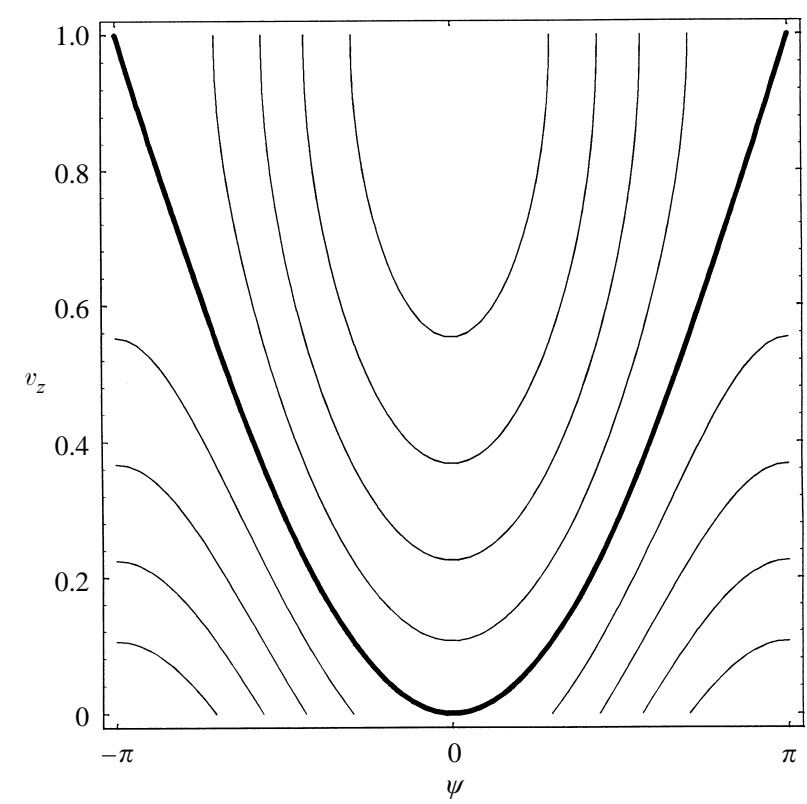

Figure 1. Phase-space portrait of the non-relativistic Hamiltonian for $\epsilon=0.5$ and $f=1$. The separatrix is shown by the thick curve.

With that, we can compute the wave amplitude $\epsilon_{\mathrm{sep}, \mathrm{nr}}$ for which the lower separatrix is just tangent to the $v_{z, n r}=0$ axis - this results in the soliton-like acceleration for particles launched with $v_{z, \mathrm{nr}, 0}=0$ and $\psi_{\mathrm{nr}}=0$; in agreement with the calculations of Kuo and Lee (1994), we find

$$
\epsilon_{\mathrm{sep}, \mathrm{nr}}=\frac{1}{2} f .
$$

Let us now turn to the relativistic case, starting with an initial look at the corresponding phase space. In order to make the phase-space analysis clearer and more similar to the non-relativistic case, it is worthwhile to rewrite the Hamiltonian (2.5) as a function of $v_{z}$ instead of $P_{z}$. From the relation $P_{z}=\gamma v_{z}$, we have

$$
P_{z}=v_{z}\left[\frac{1+2 \epsilon^{2}(1-\cos \psi)}{1-\left(v_{z}\right)^{2}}\right]^{1 / 2} .
$$

Using the above equation in (2.5), we find the desired function. In Fig. 2, we show a contour plot of $\mathcal{H}=\mathcal{H}\left(v_{z}, \psi\right)$ for $\epsilon=0.5$ and $f=0.8$. Although the phase space differs substantially from the non-relativistic one, the presence of trapped and untrapped orbits separated by a (highlighted) separatrix trajectory can be still appreciated.

In the fully relativistic case we can obtain an expression equivalent to (3.2): it reads

$$
\epsilon_{\mathrm{sep}}=\frac{f}{2\left(1-f^{2}\right)^{1 / 2}} .
$$

In Fig. 3, we plot $\epsilon_{\text {sep }}$ and $\epsilon_{\text {sep,nr }}$ versus $f$. The solid line represents values obtained from the relativistic formalism and the dashed line those obtained from the nonrelativistic one. As could be expected, in the relativistic case $\epsilon_{\text {sep }}$ diverges as $f \rightarrow 1$. In this limit, the maximum longitudinal momentum rapidly grows with $v_{z, \max } \rightarrow 1$, and infinite wave amplitudes are required. 


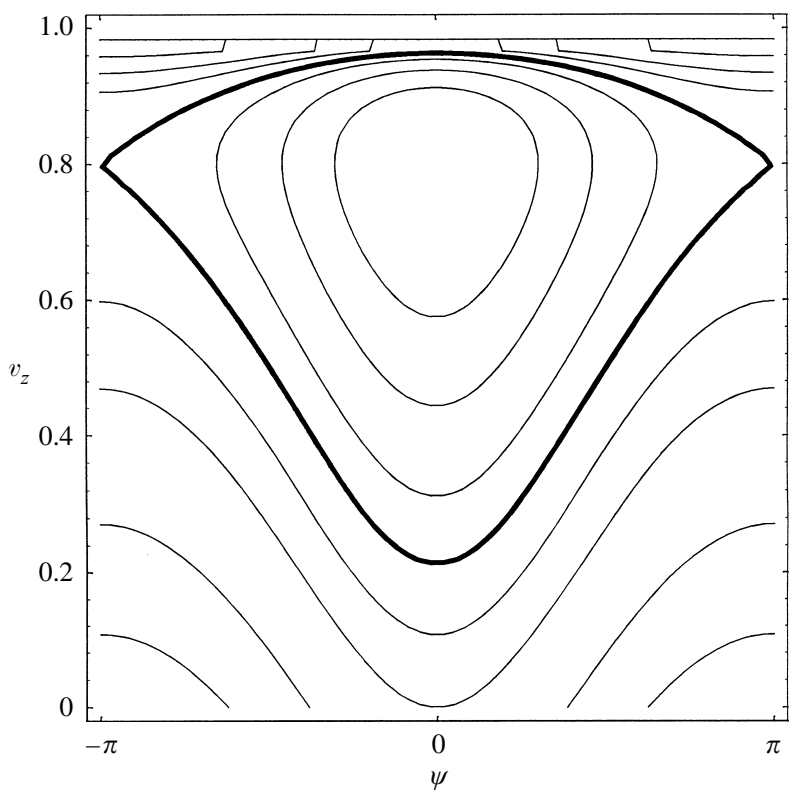

Figure 2. Phase-space portrait of the relativistic Hamiltonian for $\epsilon=0.5$ and $f=0.8$. The separatrix is shown by the thick curve.

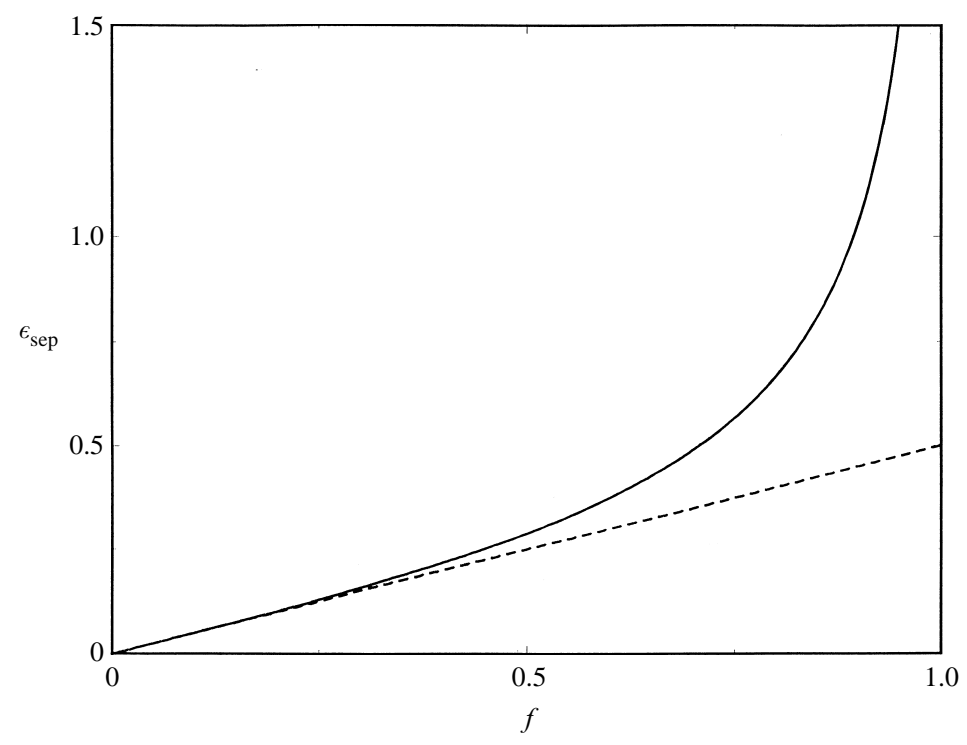

Figure 3. Wave amplitude $\epsilon_{\text {sep }}$ versus the normalized wave phase velocity $f$. The solid (dashed) line represents the relativistic (non-relativistic) case.

\subsection{Generic case}

Given that we have to work with finite fields, we immediately rule out the solitonlike acceleration for $f=1$. It is fairly intuitive, as can be checked with little calculation, that larger wave amplitudes imply larger acceleration. However, a subtler question yet to be answered is the following: for a fixed and finite value of the wave 
amplitude, what is the value of $f$ creating the largest acceleration? Or, in other words: what is the optimal value of $f$ ? We shall address this issue from now on.

Recalling that for finite wave amplitudes the $f=1$ separatrix cannot touch the $v_{z}=0$ axis, particles launched with that value of $f$, at $v_{z, 0}=0$ and $\psi_{0}=0$, will describe untrapped orbits. If we decrease $f$, the separatrix also starts to move lower down and get closer to $v_{z}=0$, until, at $f=f_{\text {sep }}$, it touches that axis. At this moment, initially low-energy particles undergo soliton-like acceleration similarly as described in the previous subsection. Further decrease of $f$ ultimately causes these low-energy particles to become trapped in the wave field.

Let us proceed to an explicit calculation of the maximum velocity excursions allowed in the trapped and untrapped cases, recalling that particles with $v_{z, 0}=0$ and $\psi_{0}=0$ always evolve in time, preserving $\mathcal{H}=1$.

As can be seen from Fig. 2, untrapped particles undergo maximum excursion at $\psi=-\pi$. At this location, using both (2.5) and (3.3), and the fact that $\mathcal{H}=1$, we find a quadratic equation for $v_{z}$ :

$$
\left(1+A f^{2}\right) v_{z}^{2}-2 A f v_{z}+A-1=0,
$$

where $A \equiv 1+4 \epsilon^{2}$. The existence of two roots for $v_{z}$ is natural because the system posseses two orbits with $\mathcal{H}=1$ : one below the lowest separatrix and the other above the highest. The solution describing the velocity excursion in which we are interested is, of course, the one with the lowest speed:

$$
v_{z, \max }^{\mathrm{untr}}=\frac{A f-\left[A^{2} f^{2}-\left(1+f^{2} A\right)(A-1)\right]^{1 / 2}}{1+f^{2} A} .
$$

When the discriminant of (3.5) vanishes, both roots coalesce at $v_{z, \text { max }}^{\mathrm{untr}}=f_{\mathrm{sep}}=v_{z}^{u}$, where $f_{\text {sep }}$ is obtainable as a function of $\epsilon$ as in (3.4). At this point, particles with $v_{z, 0}=0$ and $\psi_{0}=0$ find themselves orbiting along the separatrix of the system. For $f<f_{\text {sep }}$, solutions of (3.5) are imaginary; this means that particles become trapped, no longer reaching $\psi=-\pi$.

Let us proceed with the investigation, considering the trapped case for $f<f_{\text {sep }}$. From Fig. 2, we readily note that trapped particles undergo maximum acceleration at $\psi=0$. Then, using (2.5) and (3.3) with $\psi=0$, and the fact that $\mathcal{H}=1$, we find

$$
v_{z, \max }^{\mathrm{tr}}=\frac{2 f}{1+f^{2}},
$$

which is the maximum trapped velocity excursion for initially low-energy particles.

In Fig. 4 we gather our results (3.6) and (3.7), and plot the maximum velocity excursion $v_{z, \max }$, versus $f$ for $\epsilon=0.5$. The discontinuity in the curve appears at $f=f_{\text {sep }}$, where trajectories change their topological character from trapped to untrapped. It is apparent that the curve is not monotonic, presenting just to the left of the discontinuity an optimal $f, f_{\text {opt }}$, leading to the largest possible $v_{z}$ for a fixed wave amplitude $\epsilon$. It is thus seen that effective acceleration depends on a judicious choice of $f$. In other words, contrarily to what could be thought initially, for finite wave fields the most effective acceleration does occur for subluminal waves, $f<1$, and not at $f=1$.

In order to get a clearer idea of the advantage of operating an accelerator with optimal $f$ instead of the soliton-like acceleration, in Fig. 5 we compare the maximum energization $\gamma_{\max }$ for the optimal $f_{\text {opt }}$ lying an infinitesimal amount below $f_{\text {sep }}$ (solid line), and the corresponding $\gamma$ calculated for $f=f_{\text {sep }}$ (dashed line). For 


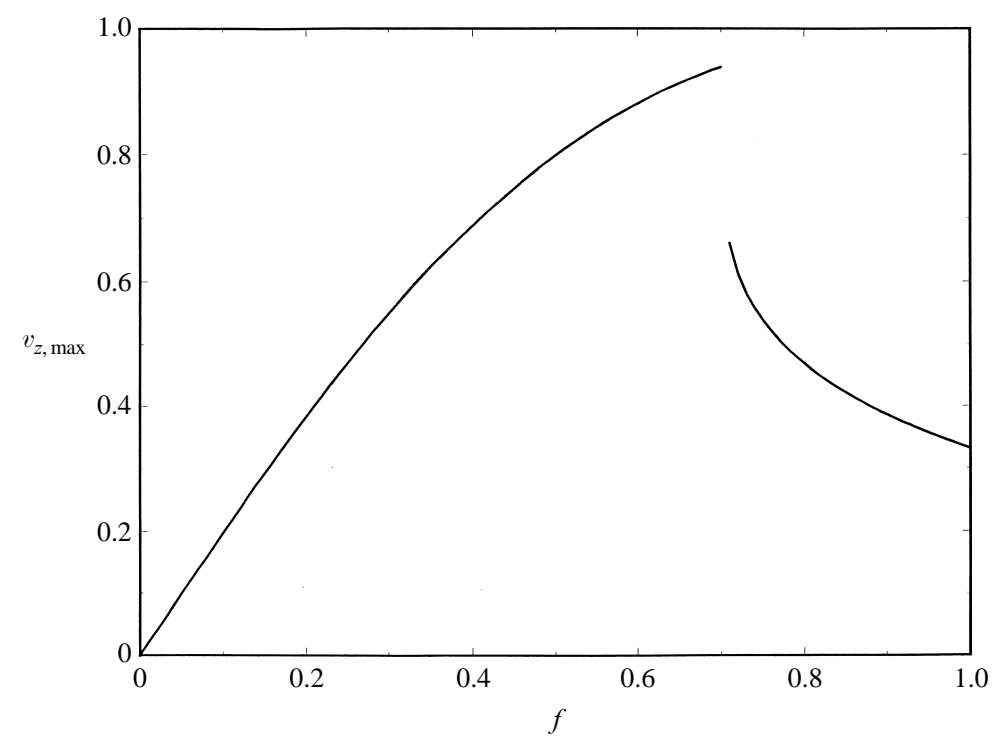

Figure 4. Maximum fully relativistic longitudinal speed $v_{z, \max }$ versus $f$ for $\epsilon=0.5$.

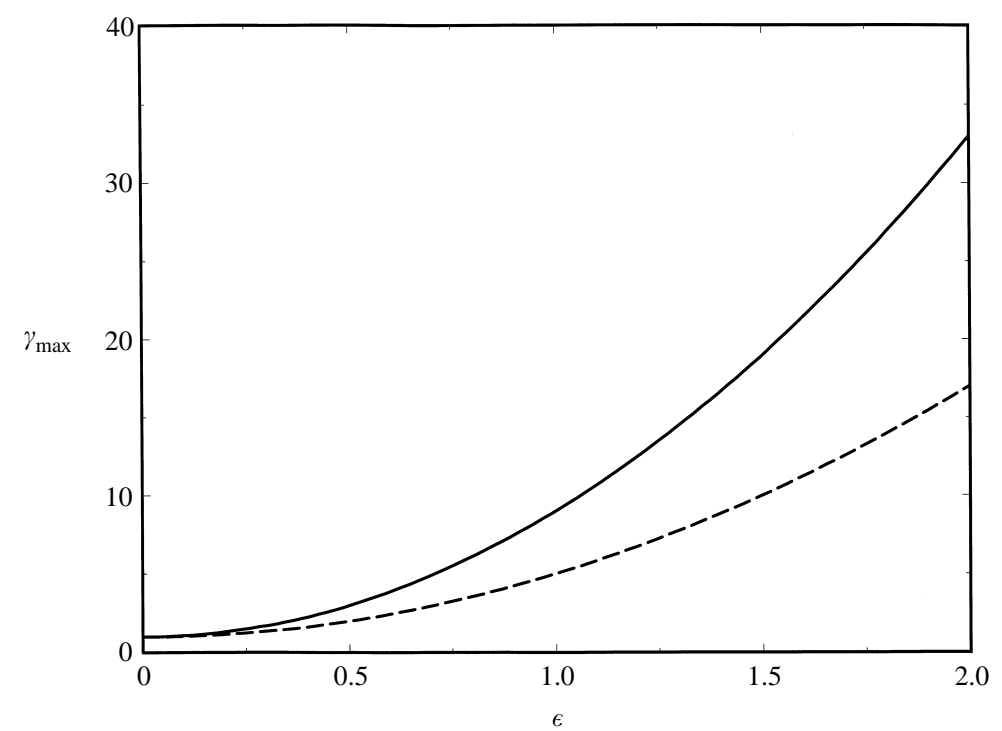

Figure 5. Maximum energy allowed $\gamma_{\max }$ versus the wave amplitude $\epsilon$ for $f=f_{\text {opt }}$ (solid line) and $f=f_{\text {sep }}$ (dashed line).

initially low-energy particles, the relativistic factor is computed as a function of $v_{z}$ from $(2.5)$, with $\mathcal{H}=1$, and from the relation $P_{z}=\gamma v_{z}$ as

$$
\gamma=\left(1-f v_{z}\right)^{-1}
$$

$f_{\text {sep }}$ is to be expressed as a function of $\epsilon$ from (3.4). Once again, it is apparent from the figure that higher energization is obtained if one is dealing with $f=f_{\text {opt }}$. 


\section{Summary}

The possibility of reaching speeds of the order of the speed of light $c$ makes it necessary to include relativistic effects in the interaction of charged particles and large-amplitude circularly polarized electromagnetic waves.

Taking into account full relativistic effects via a Hamiltonian formulation of the problem, we have found that wave amplitudes necessary to drive soliton-like acceleration along the lines proposed by Kuo and Lee (1994) diverge when $f \rightarrow 1$. However, we have shown that for finite field amplitudes the accelerating process can still be very efficient if one uses an optimal $f$ satisfying $f_{\text {opt }}<1$.

The particle energization could be seen as an inverse Čerenkov effect where lowenergy particles absorb energy from an electromagnetic wave in a medium where the phase velocity is smaller than $c$.

For $\epsilon \approx 0.5$ and $\omega \approx 10 \mathrm{GHz}$, which means electric fields of the order of $10^{6} \mathrm{~V} \mathrm{~m}^{-1}$ - typical of moderate laboratory waves - and $f=f_{\text {opt }}$, we find for the maximum longitudinal speed allowed $v_{z, \max } \sim 0.93 \mathrm{c}$.

\section{Acknowledgements}

We should like to thank an anonymous referee for valuable suggestions. This work was partially supported by Financiadora de Estudos e Projetos (FINEP) and Conselho Científico e Tecnológico (CNPq), Brasil.

\section{References}

Chen, C. 1992 Phys. Rev. A46, 6654.

Davidson, R. C., Yang, Y.-T. and Aamodt, R. E. 1989 J. Plasma Phys. 41, 405.

Friedland, L. 1994 Phys. Plasmas 1, 421.

Kuo, S. P. and Lee, M. C. 1994 J. Plasma Phys. 52, 339.

Pakter, R., Schneider, R. and Rizzato, F. B. 1993 Phys. Rev. E47, 3787.

Pakter, R., Schneider, R. and Rizzato, F. B. 1994 a Phys. Rev. E49, 1594.

Pakter, R., Corso, G., Caetano, T. S., Dillenburg, D. and Rizzato, F. B. $1994 b$ Phys. Plasmas 1, 4099 .

Sprangle, P., Vlahos, L. and Tang, C. M. 1983 IEEE Trans. Nucl. Sci. 30, 3177.

Von Laven, S., Brancum, J., Golub, J., Layman, R. and Walsh, J. 1982 Appl. Phys. Lett. 41 , 408. 